\title{
The statistical significance of the North-South asymmetry of solar activity revisited
}

\author{
M. Carbonell ${ }^{1}$, J. Terradas ${ }^{2}$, R. Oliver ${ }^{2}$, and J. L. Ballester ${ }^{2}$ \\ 1 Departament de Matemàtiques i Informàtica, Universitat de les Illes Balears, 07122 Palma de Mallorca, Spain \\ e-mail: marc.carbonell@uib.es \\ 2 Departament de Física, Universitat de les Illes Balears, 07122 Palma de Mallorca, Spain \\ e-mail: [jaume.terradas; ramon.oliver; joseluis.ballester]@uib.es
}

Received 4 June 2007 / Accepted 26 July 2007

\section{ABSTRACT}

\begin{abstract}
Aims. Many studies of the North-South asymmetry of solar activity and its features have been performed. However, most of these studies do not consider whether or not the asymmetry of the time series under consideration is statistically significant. If the asymmetry is statistically insignificant, any study about its behavior is meaningless. Here, we discuss the difficulties found when trying to assess the statistical significance of the North-South asymmetry (hereafter SSNSA) of the most usually considered time series of solar activity.

Methods. We distinguish between solar activity time series composed of integer or non-integer and dimensionless data, or composed of non-integer and dimensional data. For each of these cases, we discuss the most suitable statistical tests which can be applied and highlight the difficulties in obtaining valid information about the statistical significance of solar activity time series.

Results. Our results suggest that, apart from the need to apply suitable statistical tests, other effects such as data binning, the considered units and the need, in some tests, to consider groups of data, substantially affect the determination of the statistical significance of the asymmetry.

Conclusions. The assessment of the statistical significance of the N-S asymmetry of solar activity is difficult and an absolute answer cannot be given, since many different effects influence the results given by the statistical tests. The quantitative results about the statistical significance of the N-S asymmetry of solar activity provided by different authors, as well as studies of its behaviour, must be considered with care because they depend on the chosen values of different parameters or on the considered units.
\end{abstract}

Key words. Sun: activity - methods: data analysis - methods: statistical

\section{Introduction}

The North-South (N-S) asymmetry of solar activity has been the subject of many studies using different features of solar activity. Some of the most important features considered have been: the number of flares (Roy 1977; Garcia 1990; Verma 1987, 1992; Li et al. 1998; Temmer et al. 2001), the flare index (Knoška 1985; Ataç \& Özgüç 1996, 1998; Joshi \& Joshi 2004), the sunspot number and sunspot areas (Newton \& Milson 1955; Waldmeier 1957, 1971; Roy 1977; Swinson et al. 1986; Vizoso \& Ballester 1990; Schlamminger 1991; Verma 1992; Yi 1992; Carbonell et al. 1993; Verma 1993; Oliver \& Ballester 1994; Pulkkinen et al. 1999; Li et al. 2000; Li et al. 2002; Vernova et al. 2002; Temmer et al. 2002; Li et al. 2003; Knaack et al. 2004; Temmer et al. 2006), the photospheric magnetic flux (Rabin et al. 1991; Knaack et al. 2004) and the number of solar active prominences (Joshi 1995; Verma 2000).

There is an important solar activity feature, the photospheric magnetic flux, for which the behavior of the N-S asymmetry has been also studied. Howard (1974), using magnetic flux data from Mt. Wilson, analyzed the period between 1967 and 1973; Rabin et al. (1991) using magnetic flux data from Kitt Peak, studied the period between 1975 and 1987; Knaack et al. (2004) have used Kitt Peak data about photospheric magnetic flux density to study the time interval between 1975 and 2003; Song et al. (2005) have used Kitt Peak data, between 1978 and 2002. However, of all these authors only Song et al. (2005) have estimated the statistical significance of the North-South asymmetry (hereafter SSNSA) of the photospheric magnetic flux time series under consideration, applying the binomial distribution, and so most of the conclusions of the rest of studies about the N-S asymmetry of photospheric magnetic flux have been obtained by visual inspection of the plot of the asymmetry versus time. Taking into account the fundamental role of the magnetic flux in solar activity, a quantitative assessment of its N-S asymmetry should be of great interest.

Before studying the behavior of the N-S asymmetry, the most important point, sometimes forgotten, is to assess the SSNSA of the time series under consideration. The most straightforward way to determine the SSNSA is by means of the binomial distribution (Li et al. 1998; Li et al. 2003; Song et al. 2005). However, other statistical tests have also been used, for instance, Joshi (1995), Temmer et al. (2001) and Joshi \& Joshi (2004) have followed Leftus (1960) using a $\chi^{2}$-test to assess the SSNSA of sunspot groups, $\mathrm{H} \alpha$ flares and active prominences; Temmer et al. $(2002,2006)$ have used a paired Student's test to study the SSNSA of the hemispheric sunspot number; Ataç \& Özgüç (1996) used a sign test, introduced by Gleissberg (1947), to determine the SSNSA of the flare index, and Vizoso \& Ballester (1990) and Carbonell et al. (1993) have used Excess (Reid 1968; Wilson 1987) to obtain the SSNSA of sunspot areas. In all the cases mentioned before, the authors concluded that, to a great 
extent, the N-S asymmetry of the considered time series was statistically significant.

However, a blind application of the above statistical tests to any considered solar activity time series can lead to misleading results. The commonly considered solar activity time series have two different forms: (a) composed of integer or non-integer data; (b) composed of dimensional or dimensionless data. The first characteristic, integer or non-integer data, is relevant for the statistical tests that can be applied. In the case of integer data records any statistical test can be applied, but in the case of noninteger data one needs to choose carefully what tests can be used. Related to the second characteristic, dimensional or dimensionless data, this raises two interesting problems when dimensional data are considered: (a) how does a change of units affect the statistical significance? (b) Is it possible to find a statistical test whose results are independent of the considered units?

Here, we search for quantitative conclusions about the SSNSA of the most often considered solar activity time series. To this end, we analyse the results obtained after the application of different statistical tests to solar activity time series made of integer and dimensionless data, and the effects on the SSNSA induced by data binning and point out the problems encountered when trying to assess the SSNSA of non-integer and dimensional solar activity time series. We perform these analysis since many conclusions about the behaviour of the N-S asymmetry of solar activity have been extracted without a proper quantitative evaluation of its statistical significance, or without considering whether a definitive answer about the SSNSA can be obtained.

\section{Data and methods}

\subsection{Data}

The different solar activity time series analyzed in our study are:

- The daily north and south international sunspot number (1992-2004), which can be downloaded from http://www . ngdc . noaa.gov/stp/SOLAR/ftpsunspotnumber.html, with 4718 data points.

- The daily north and south hemispheric sunspot numbers (1945-2004), compiled by Temmer et al. (2006), which can be downloaded from http: //cdsweb.u-strasbg.fr/ cgi-bin/qcat? J/A+A/447/735, with 21915 data points.

- The yearly north and south number of active prominences (1957-1998), obtained from Verma (2000), with 42 data points.

- The daily north and south X-ray flares (1975-2006), detected by the GOES satellites, which can be downloaded from http://www.ngdc.noaa.gov/stp/SOLAR/ ftpsolarflares.html, with 11323 data points.

- The daily number of north and south solar flare index (1976-2004) compiled in the Kandilli Observatory, which can be downloaded from http://wWw.ngdc.noaa.gov/ stp/SOLAR/ftpsolarflares.html, with 10227 data points.

- The monthly north and south sunspot area between May 1874 and January 2004 compiled by D. Hathaway, and which can be downloaded from http://science. msfc.nasa.gov/ssl/pad/solar/greenwch.htm, with 1557 data points.

- The north and south NSO/Kitt Peak averaged photospheric magnetic flux density corresponding to Carrington rotations 1625-2007 (February 1975-August 2003), with 383 data points.
- The north and south total magnetic flux from Mt. Wilson Observatory corresponding to Carrington rotations 1511-2013 (September 5, 1966-August 26, 2004), with 502 data points.

\subsection{Preliminary considerations}

Usually, the application of a statistical test is based on the hypothesis of independence of experiments. However, when we consider the N-S asymmetry of solar activity, the data records of many of the considered time series are not independent. For instance, if we consider daily sunspot areas, magnetic flux, solar prominences, sunspot number, etc. the values of these quantities today are not independent of the values yesterday, in particular, daily sunspot areas are correlated with a typical correlation time of about 7 days (Oliver \& Ballester 1995). Then, strictly speaking, solar activity does not satisfy the above hypothesis, and, for this reason, the application of statistical tests to determine the SSNSA is not appropriate. Another consideration of interest is related to the characteristics of the studied solar activity time series which can be classified as composed of integer and dimensionless data, such as the International sunspot number, the number of X-ray flares and the number of solar active prominences, or composed of non-integer and dimensional data, such as the $\mathrm{H}_{\alpha}$ flare index, sunspot areas, Mount Wilson total magnetic flux and Kitt Peak averaged magnetic flux density. This second consideration is of paramount importance since it constrains the statistical tests that can be applied to the above time series. The third consideration is that in all the studies about the $\mathrm{N}-\mathrm{S}$ asymmetry we only consider the solar activity corresponding to the visible hemisphere.

\subsection{Statistical tests for dimensionless time series}

To assess the SSNSA of solar activity time series, we distinguish between integer and dimensionless time series, non-integer and dimensionless time series, and non-integer and dimensional time series. In the case of integer and dimensionless time series we have applied the following statistical tests:

1. Binomial distribution: when the data records of the considered time series are integer numbers, the binomial formula (Larson 1982) can be used to compute the probability of obtaining any particular distribution of $n$ objects into two classes. In our case, these two classes correspond to North and South hemispheres $(N$ and $S$ ). The binomial formula is given by

$P(r)=\frac{n !}{r !(n-r) !} p^{r}(1-p)^{n-r}$

where $n$ is the number of objects in both classes, $r$ is the number of objects in a particular class, and $p$ is the probability associated with that particular class, in our case 0.5 . An alternative way (Wilson 1987) is to describe the probabilities using the difference $d=|r-(n-r)|$ with $P(d)=2 \cdot P(r)$ for all $P(r)$ except when $d=0$, and $P(\geq d)$ is given by

$P(\geq d)=\sum_{i=d}^{n} P(i)$.

In general, when $P(\geq d)>10 \%$ this implies a statistically insignificant result; when $5 \%<P(\geq d)<10 \%$ it is marginally significant; when $1 \%<P(\geq d)<5 \%$ we have a statistically significant result and when $P(\geq d)<1 \%$ the result is highly significant. 
Table 1. Surface expressed in $\mathrm{dm}^{2}$. The labels of the columns, from left to right, correspond to the normal distribution approximation to the binomial distribution, to the Pearson's chi-square test, to the Excess, and to the binomial distribution. The rows, from top to bottom, correspond to a highly statistically significant result, a statistically significant result, a marginally significant result, and a statistically insignificant result. The numbers shown in each column correspond to the number of events and its corresponding percentage with respect to the data records of the considered time series.

\begin{tabular}{cccc}
\hline \hline ND & $\chi^{2}$ & $E$ & $P(\mathrm{~d})$ \\
\hline $7817(93.6 \%)$ & $7749(92.8 \%)$ & $7693(92.1 \%)$ & $7728(92.6 \%)$ \\
$165(1.9 \%)$ & $163(1.9 \%)$ & $170(2.0 \%)$ & $158(1.9 \%)$ \\
$91(1.1 \%)$ & $70(0.8 \%)$ & $180(2.1 \%)$ & $85(1.0 \%)$ \\
$272(3.2 \%)$ & $363(4.3 \%)$ & $302(3.6 \%)$ & $374(4.5 \%)$ \\
\hline
\end{tabular}

2. Excess: An approximation to compute $P(\geq d)$ is the Excess (Reid 1968; Wilson 1987), which is a measure proportional to the uncertainty, computed as $d(n / 2)^{-1 / 2}$, where $d=|N-S|$ is the positive difference between the two hemispheres and $n$ is the total quantity corresponding to both hemispheres. The equivalence of Excess and $P(\geq d)$ is given by: Excess $<2$ with $P(\geq d)>10 \% ; 2<$ Excess $<3$ with $5 \%<P(\geq d)<10 \% ; 3<$ Excess $<4$ with $1 \%<P(\geq d)<5 \%$ and $4<$ Excess with $P(\geq d)<1 \%$.

3. Normal approximation to the Binomial distribution: Another way to study the statistical significance of an asymmetry time series is to consider the normal distribution as an approximation of the binomial distribution (Larson 1982). In this case, the normal distribution has the same mean $\mu=n p$ and standard deviation $\sigma=\sqrt{n p(1-p)}$ as the binomial distribution. Then, we have considered the standard normal distribution with $p=0.5$

$Z=\frac{X-\mu}{\sigma}=\frac{N-S}{\sqrt{(N+S)}}$.

To test the SSNSA of our time series, we have counted the number of events in which the statistic $Z$ lies in different intervals $0 \leq Z \leq 0.01$ or $0.99 \leq Z \leq 1$, which indicate that the asymmetry is highly significant.

4. Pearson's chi-square test $\left(\chi^{2}\right)$ : For this goodness of fit computation (Larson 1982), the data are divided into $k$ classes and the test statistic is defined as

$\chi^{2}=\sum_{i=1}^{k} \frac{\left(O_{i}-E_{i}\right)^{2}}{E_{i}}$,

where $O_{i}$ represents the observed frequency and $E_{i}$ the expected (theoretical) frequency, asserted by the null hypothesis $H_{0}$. In our case, $H_{0}$ is that the observed data follow a binomial distribution with $p=1 / 2$. So our statistic is:

$\chi^{2}=\sum_{i=1}^{2} \frac{\left(N_{i}-n p_{i}\right)^{2}}{n p_{i}}=\frac{(N-S)^{2}}{N+S}$.

The obtained test statistic is then compared with a chi-square distribution $\chi_{1, \alpha}^{2}$ with one degree of freedom, since we only consider two classes. The significance level $\alpha$ is taken in such a way that when $\chi^{2} \leq 0.01$ (highly significant asymmetry), $0.01<\chi^{2} \leq 0.05$ (significant asymmetry), $0.05<$ $\chi^{2} \leq 0.1$ (marginal asymmetry); $0.1<\chi^{2} \leq 1$ (insignificant asymmetry).

Probably, the most suitable test to be applied in order to determine the SSNSA is the binomial distribution test. However, this test can only be applied when the data elements of the considered time series are integers, which constrains its applicability to time series of solar activity made of the number of events happening in each solar hemisphere. On the other hand, this test has to be used properly, as we now illustrate by considering two synthetic time series corresponding to the North and South hemispheres, respectively. Let us assume that we construct two time series composed of 105 data points taken, for instance, at consecutive Carrington rotations. In the Northern hemisphere, the first 63 data points have a value of 6 while the remaining 42 have a value of 4 ; in the Southern hemisphere the first 63 data points have a value of 4 and the remaining 42 have a value of 6 . Song et al. (2005) consider that for 63 rotations the northern hemisphere has been dominant and they take this quantity as $d$ while $n$ has the value of 105 . In this way the result of the test is independent of the values of quantities in the Northern and Southern hemispheres. Computing $P(d)$ gives a value of 0.0252 (2.5\%) which suggests a strong statistical significance. However, a proper application of the binomial distribution is based on determining the SSNSA of each pair of values (North and South) corresponding to the same Carrington rotation, evaluating, finally, the number of pairs whose SSNSA is highly significant. Then, after applying this procedure to the above considered time series we obtain that $P(d)$ is greater than $10 \%$ in all of the 105 cases. This means that the SSNSA of these synthetic data points is insignificant in all the Carrington rotations as should be expected from the small difference between the Northern and Southern hemisphere values.

In the case of non-integer and dimensionless time series, only the tests Excess, Normal approximation to the Binomial distribution, and Pearson's chi-square test can be applied.

\subsection{Statistical tests for non-integer and dimensional time series}

When the data are dimensional another problem appears related to the considered units. In solar activity time series, the accuracy of the non-integer and dimensional data is determined by the measurement process, so the data are truncated after some decimal places. One way to obtain integer data is to modify the units of the considered time series, and to apply the statistical tests described in the Sect. 2.3. Then, an interesting experiment is to consider what happens to the statistical significance when the units are modified. A simple way to do this is to generate two synthetic time series, corresponding to Northern and Southern hemispheres, made of non-integer and dimensional data. We have chosen two time series composed of data records representing surfaces expressed in square meters up to two decimal places. These non-integer data can be transformed to integer data by multiplying by $10^{2}$ to obtain surfaces in $\mathrm{dm}^{2}$, or by multiplying by $10^{4}$ to obtain surfaces in $\mathrm{cm}^{2}$. To the resulting time series we have applied the tests of the previous section and the results are shown in Tables 1,2 which point out that, when going 
from $\mathrm{dm}^{2}$ to $\mathrm{cm}^{2}$, the SSNSA changes and increases. These results suggest that transforming non-integer dimensional data to integer data by changing the units modifies the statistical significance. These results point out a difficult problem because when dealing with dimensional time series the SSNSA will depend on the considered units, at least when the above tests are used. Our problem now is to find a statistical test applicable to non-integer and dimensional time series, and whose results are independent of the considered units.

\subsection{The Student's t-test}

A suitable test satisfying the above conditions is the paired Student's t-test (Larson 1982). The characteristic statistic $\hat{t}$ is expressed as

$$
\hat{t}=\frac{\sum D_{i} / n}{\sqrt{\frac{\sum D_{i}^{2}-\left(\sum D_{i}\right)^{2} / n}{n(n-1)}}},
$$

where $D_{i}$ is the difference of paired values and $n$ represents the number of elements that we have in each of the groups in which we split the corresponding time series, $n-1$ being the number of degrees of freedom. Once the statistic $\hat{t}$ has been calculated, and taking into account the degrees of freedom, it is compared with $\hat{t}_{n-1}, \alpha$ given in statistical tables on a previously chosen probability $\alpha$. The important feature of this test is that when the units are changed, the same factor appears in both the numerator and denominator, and the value of the $\hat{t}$-statistic is not modified. This can be ckecked using the surface time series, in $\mathrm{m}^{2}, \mathrm{dm}^{2}$, and $\mathrm{cm}^{2}$, considered in the previous section. Then, taking $n=30$ we obtain the same results for the three time series i.e. the value of the statistic $\hat{t}$ is: $>0.99$ for $32.05 \%$ of the groups; between 0.95 and 0.99 for $18.82 \%$ of the groups; between 0.9 and 0.95 for $14.70 \%$ of the groups; and smaller than 0.9 for $34.11 \%$ of the groups. Taking this feature into account, the Student's test can be very useful for the determination of the SSNSA of non-integer and dimensional time series.

\section{Results}

\subsection{Results for integer and dimensionless time series}

The statistical tests mentioned in Sect. 2.3 have been applied to the time series corresponding to the daily international sunspot number, the yearly number of solar active prominences and the daily number of X-ray flares, and the obtained results are displayed in Tables 3-5. In Table 3, the SSNSA corresponding to the daily international sunspot number is shown and a good agreement between the results obtained with the different tests can be seen. Also, the results show that the N-S asymmetry of the time series is only highly significant or statistically significant on about $50-60 \%$ of the considered days. In Table 4 , the results obtained for the yearly number of solar active prominences are shown. These results show a striking agreement in the statistical significance between the different tests, and suggest that the N-S asymmetry is highly significant in about $85 \%$ of the considered years. In Table 5, the results for the daily number of solar Xray flares are shown. These results indicate a very low statistical significance (between 3-7\% of the days) for the N-S asymmetry for this time series, which suggests that this solar activity feature displays no significant N-S asymmetry.

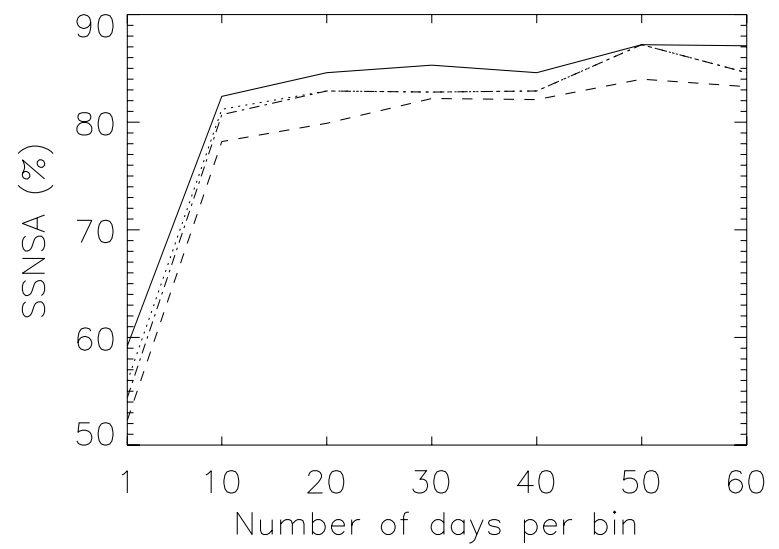

Fig. 1. SSNSA of the international sunspot number time series versus the number of days per bin for the binomial distribution (dash-dot line); Excess (dashed line); normal approximation to the binomial distribution (solid line); and Pearson's chi-square test (dotted line).

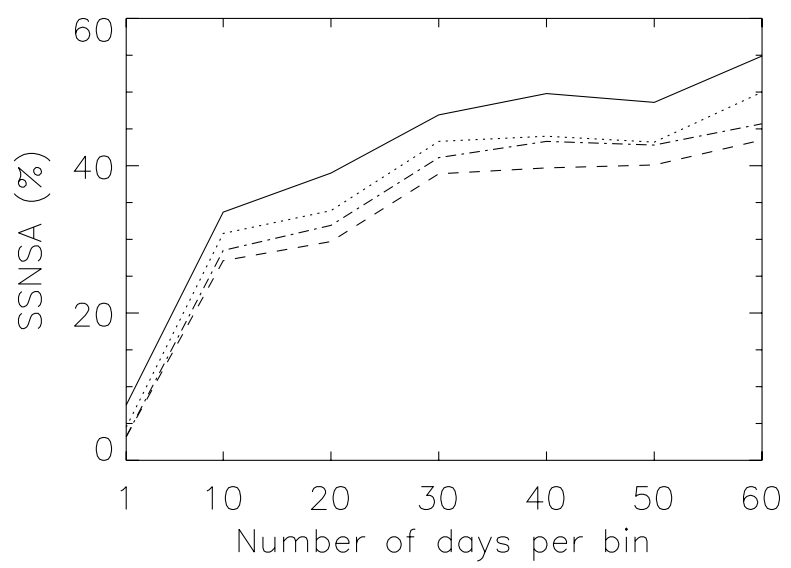

Fig. 2. SSNSA of the number of X-ray flare time series versus the number of days per bin for the binomial distribution (dash-dot line); Excess (dashed line); normal approximation to the binomial distribution (solid line); and Pearson's chi-square test (dotted line).

\subsubsection{Effect of data binning}

We study the effect of binning data on the statistical significance. We have binned the time series corresponding to the international sunspot number and X-ray flare in bins of 10, 20, 30, 40, 50 and 60 days. The same statistical tests as in previous section have been applied to these new time series and in Figs. 1, 2 the SSNSA versus the number of days per bin is plotted. Binning data modifies the statistical significance, and the general trend is an increase of the SSNSA. If one starts from two north and south daily time series and computes the SSNSA, the obtained results would be different from those obtained by binning the times series for Carrington rotation and computing, again, the statistical significance. In order to compute a meaningful statistical asymmetry, what is the appropiate data binning, if any, to be considered?

\subsection{Results for non-integer and dimensionless time series}

The only considered solar activity time series whose data records are non-integer and dimensionless is that of hemispheric sunspot numbers. To this time series we have applied the tests given in Sect. 2.5. The results are shown in Table 6, which shows that there is a strong agreement between the statistical significances 
Table 2. Surface expressed in $\mathrm{cm}^{2}$. Columns, rows and numbers in the rows have the same meaning as in Table 1.

\begin{tabular}{cccc}
\hline \hline ND & $\chi^{2}$ & $E$ & $P(\mathrm{~d})$ \\
\hline $8296(99.4 \%)$ & $8293(99.3 \%)$ & $8288(99.3 \%)$ & $8293(99.3 \%)$ \\
$16(0.2 \%)$ & $17(0.2 \%)$ & $14(0.1 \%)$ & $17(0.2 \%)$ \\
$8(0.1 \%)$ & $2(0.02 \%)$ & $15(0.1 \%)$ & $2(0.02 \%)$ \\
$25(0.3 \%)$ & $33(0.4 \%)$ & $28(0.3 \%)$ & $33(0.4 \%)$ \\
\hline
\end{tabular}

Table 3. Daily international sunspot number. Columns, rows and numbers in the rows have the same meaning as in Table 1.

\begin{tabular}{cccc}
\hline \hline ND & $\chi^{2}$ & $E$ & $P(\mathrm{~d})$ \\
\hline $2608(59.2 \%)$ & $2463(55.9 \%)$ & $2307(52.3 \%)$ & $2398(54.4 \%)$ \\
$451(10.2 \%)$ & $410(9.3 \%)$ & $449(10.2 \%)$ & $394(8.9 \%)$ \\
$220(5.0 \%)$ & $186(4.2 \%)$ & $440(10 \%)$ & $198(4.5 \%)$ \\
$1128(25.6 \%)$ & $1348(30.6 \%)$ & $1211(27.5 \%)$ & $1417(32.2 \%)$ \\
\hline
\end{tabular}

Table 4. Yearly number of active solar prominences. Columns, rows and numbers in the rows have the same meaning as in Table 1.

\begin{tabular}{cccc}
\hline \hline ND & $\chi^{2}$ & $E$ & $P(\mathrm{~d})$ \\
\hline $36(85.7 \%)$ & $36(85.7 \%)$ & $35(83.3 \%)$ & $35(83.3 \%)$ \\
$0(0 \%)$ & $1(2.4 \%)$ & $1(2.4 \%)$ & $1(2.4 \%)$ \\
$2(4.8 \%)$ & $0(0 \%)$ & $2(4.8 \%)$ & $0(0 \%)$ \\
$4(9.5 \%)$ & $6(14.3 \%)$ & $4(9.5 \%)$ & $6(14.3 \%)$ \\
\hline
\end{tabular}

obtained from the different tests. Furthermore, the results indicate that the N-S asymmetry of hemispheric sunspot numbers is highly significant in about $60 \%$ of the considered days.

\subsection{Results for non-integer and dimensional time series}

Here, the Student's t-test described in Sect. 2.5 has been applied. We have chosen $\alpha=0.01$ and $n=30$, which means that for sunspot areas we have considerered groups of 30 months; for the $\mathrm{H}_{\alpha}$ flare index, groups of 30 days; and for the Mount Wilson total magnetic flux and the Kitt Peak averaged magnetic flux density, groups of 30 Carrington rotations. Table 7 shows the results for the SSNSA and in the case of sunspot areas, only $33 \%$ of the thirty- month groups are significant at the $99 \%$ level; in the case of the $\mathrm{H}_{\alpha}$ flare index only $32 \%$ of the thirty-day groups are significant at the $99 \%$ level; in the case of the Mount Wilson total magnetic flux only $50 \%$ of the thirty Carrington rotation groups are significant at the $99 \%$ level; and in the case of the Kitt Peak averaged magnetic flux density only $58 \%$ of the thirty Carrington rotations groups are significant at the $99 \%$ level. However, an important point to be considered when applying this test is the value chosen for $n$. We can highlight this point by considering different values for $n$, and repeating the above calculations. In Fig. 3 the SSNSA versus $n$, number of elements in each group, has been plotted, and it can be seen that the significance increases with $n$. Thus the choice of the value of $n$ is important because it determines the significance of the asymmetry, modifying it when $n$ is modified.

Another interesting situation is the case of solar activity time series showing consecutive episodes with alternate dominance between hemispheres. Assume, for instance, that we have a time series, corresponding to the Northern hemisphere, such as:

North $=60,60,60,60,60,60,2,3,4,5,6,7,60,60,60,60,60$, $60,2,3,4,5,6,7,60,60,60,60,60,60,2,3,4,5,6,7$

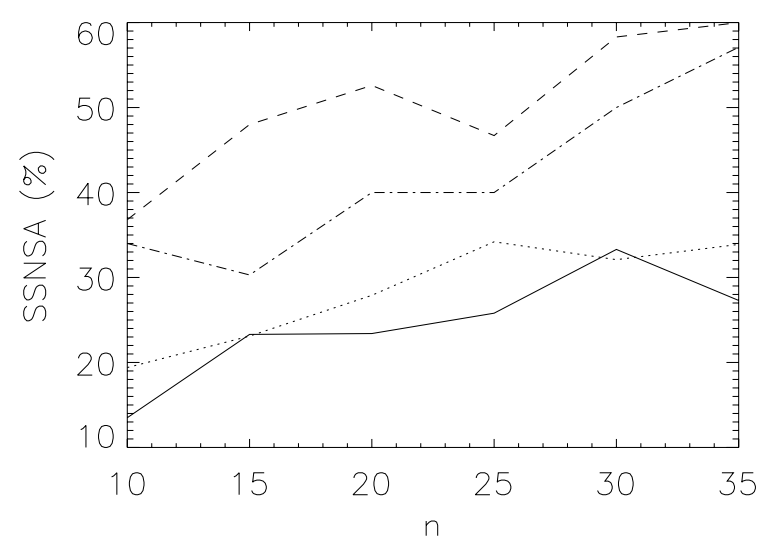

Fig. 3. Student's t-test: SSNSA of sunspot area (solid line), $\mathrm{H}_{\alpha}$ flare index (dotted line), Mount Wilson total magnetic flux (dash - dot line), and Kitt Peak averaged magnetic flux density (dashed line), versus the number $(n)$ of elements in each group.

and another one, corresponding to the Southern hemisphere, such as:

South $=1,2,3,4,5,6,60,60,60,60,60,60,1,2,3,4,5,6,60$, $60,60,60,60,60,1,2,3,4,5,6,60,60,60,60,60,60$

representing an extreme case of consecutive strong asymmetry between hemispheres. First, we take $n=6$ and determine the SSNSA between time series, obtaining that it is highly significant $(t \geq 0.99)$ for the six groups. Next, we consider $n=12$ and we determine, again, the SSNSA, obtaining that the asymmetry in this case is not significant at all $(t<0.9)$ for the 3 groups generated. This example points out the effect of the chosen $n$ since in the case $n=6$ the test detects the asymmetry between hemispheres, however, in the case $n=12$ the test is unable to detect the asymmetry since in this case the numerator of the statistic $\hat{t}$ is almost zero. Then, in extreme cases, such as the one illustrated above, a null result can be misleading since it could mean that the value of $n$ corresponds to a dominance of the Northern 
Table 5. Daily number of X-ray flares. Columns, rows and numbers in the rows have the same meaning as in Table 1.

\begin{tabular}{cccc}
\hline \hline ND & $\chi^{2}$ & $E$ & $P(\mathrm{~d})$ \\
\hline $646(7.5 \%)$ & $386(4.5 \%)$ & $277(3.2 \%)$ & $277(3.2 \%)$ \\
$1250(14.6 \%)$ & $834(9.7 \%)$ & $689(8.0 \%)$ & $439(5.1 \%)$ \\
$1100(12.8 \%)$ & $676(7.9 \%)$ & $1703(19.9 \%)$ & $394(4.6 \%)$ \\
$5568(65 \%)$ & $6668(77.8 \%)$ & $5895(68.9 \%)$ & $7454(87.0 \%)$ \\
\hline
\end{tabular}

Table 6. Daily hemispheric sunspot numbers. Columns, rows and numbers in the rows have the same meaning as in Table 1.

\begin{tabular}{cccc}
\hline \hline ND & $\chi^{2}$ & $E$ & $P(\mathrm{~d})$ \\
\hline $13025(64.83 \%)$ & $12348(61.46 \%)$ & $11547(57.46 \%)$ & - \\
$1934(9.62 \%)$ & $1694(8.43 \%)$ & $2061(10.25 \%)$ & - \\
$1076(5.35 \%)$ & $917(4.56 \%)$ & $2042(10.16 \%)$ & - \\
$4055(20.18 \%)$ & $5131(25.54 \%)$ & $4440(22.10 \%)$ & - \\
\hline
\end{tabular}

Table 7. Student's t-test. The numbers in the row define the percentage of groups of each time series that are statistically highly significant $(t \geq 0.99)$. The value of $n$ in the Student's t-test formula is 30 .

\begin{tabular}{cccc}
\hline \hline Sunspot areas & $\mathrm{H}_{\alpha}$ Flare Index & MW magnetic flux & KP magnetic flux \\
\hline $33 \%$ & $32 \%$ & $50 \%$ & $58 \%$ \\
\hline
\end{tabular}

hemisphere followed by a dominance of the Southern hemisphere, and so on. One way to avoid this problem is to consider $\left|D_{i}\right|$ instead of $D_{i}$ in the numerator of the statistic $\hat{t}$. Then, using this consideration in the calculations of the SSNSA of the above synthetic series with $n=12$, we obtain that all the groups are highly significant $(t \geq 0.99)$, as one expects due to the behaviour of the asymmetry between hemispheres.

\subsection{Dependence of the SSNSA on the solar cycle phase}

The results shown in Tables 3-7 correspond to the SSNSA of solar activity time series spanning different time intervals that cover different phases of the solar activity cycle. Due to the difference in the covered time intervals, these time series only overlap during a few solar cycles. In order to study the behaviour of the SSNSA with the phase of the solar cycle we have considered the Northern and Southern daily number of X-ray flares and the Northern and Southern daily hemispheric sunspot number time series. The first time series covers three solar cycles and the second covers six solar cycles, and they overlap during the period 1976-2004. We have split each time series as many times as possible using the following criterion: From the middle of the descending phase of one solar cycle to the middle of the ascending phase of the following solar cycle, covering the minimum of solar activity, and from the middle of the ascending phase to the middle of the descending phase of the same solar cycle, covering the maximum of solar activity. In this way, we obtained 5 and 11 shorter time series for the daily number of Northern and Southern X-ray flares and daily hemispheric sunspot numbers, respectively. Then, we applied to both time series the statistical tests of Sect. 2.5, except the binomial distribution test, because the daily hemispheric sunspot numbers is a non-integer time series. The results obtained applying the Excess test to the daily hemispheric sunspot number time series are shown in Fig. 4. This figure shows the differences between the SSNSA around the maximum and the minimum of solar activity and, although they are not large, a systematic difference appears: the SSNSA is always higher around the minimum of solar activity. It has been pointed out (Swinson et al. 1986; Vizoso \& Ballester 1990) that the North-South asymmetry of solar activity reaches very

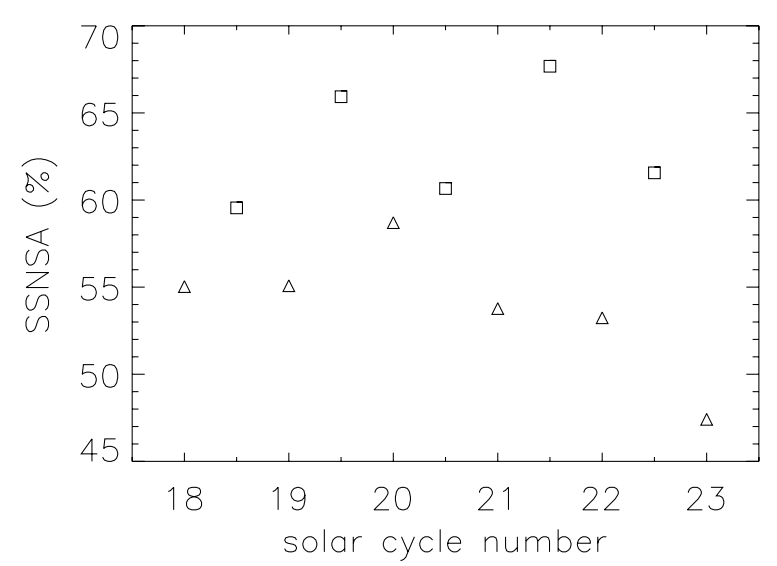

Fig. 4. SSNSA of the daily hemispheric sunspot numbers versus solar cycle. Triangles denote the SSNSA around the maximum of solar activity. Squares denote the SSNSA around the minimum of solar activity.

high values around the minimum of solar activity, thus, if only a few sunspot groups appear on the Sun and all them are in the same hemisphere, the value of the asymmetry and the SSNSA would be very high. However, around the maximum of solar activity, sunspot groups are more evenly distributed between hemispheres giving place to a lower asymmetry and SSNSA. This could be an explanation for the dependence of the SSNSA on the solar cycle phase.

A similar behaviour appears for the case of the daily number of X-ray flare time series, although in this case the SSNSA in any considered phase of the solar cycle is very low, such as can be expected due to the low SSNSA obtained when this time series is considered as a whole (see Table 5). The results obtained with the rest of tests are similar to those shown in Fig. 4.

\section{Conclusions}

We have discussed the difficulties encountered when trying to assess the SSNSA of the most common solar activity time series. We have found that in the case of integer or non-integer and dimensionless data sets several statistical tests such as the binomial 
distribution, normal approximation to the binomial distribution, chi-square test and Excess can be used, however, the obtained results strongly depend on the data binning applied. On the other hand, when non-integer and dimensional data are considered, a statistical test independent of the units can be used, the Student's t-test, but the obtained results depend again on the value chosen for the binning. Our results also suggest that there is a systematic difference between the values of the SSNSA around the maximum and the minimum of solar activity, which suggests that there is a dependence of the SSNSA on the solar cycle phase.

Taking into account these results, how can we assess the SSNSA? It seems that a definitive answer cannot be given because in one case it strongly depends on the data binning performed using, mostly, our terrestrial calendar (days, months, Carrington rotation, years, solar cycles, etc.) while solar activity does not care about it, and in the other case the answer depends on the number of elements in each considered group. In the case of integer or non-integer and dimensionless time series, the length of the bin should help to reveal physically meaningful results. Thus, for a dataset of daily values to take $n=30$ would be appropiate since this would correspond to about one solar rotation. Furthermore, if one wants to obtain some information about phases of solar activity such as the ascending or the descending branch of the solar cycle, or about the period around the maximum or the minimum of solar activity, then the length of the bin has to be chosen in agreement with the time intervals under study. These considerations could also be applied to the case of non-integer and dimensional time series because when using the Student's t-test we also need to make a choice for the value of $n$, the number of elements in each of the groups in which we split the dataset. Thus, a visual inspection of time series is not appropriate to ascertain the N-S asymmetry of solar activity time series but, on the other hand, to determine an absolute value of the SSNSA is difficult, worsened by the fact that the records of solar activity are not independent.

All the results obtained up to now on the SSNSA of different solar activity time series by different authors must be considered with care, as must be the studies performed of the behaviour of the N-S asymmetry of different solar activity features which assume that there is a real and significant asymmetry between hemispheres.

Acknowledgements. We acknowledge the National Geophysical Data Center, from whose ftp server the Kandilli flare index and international sunspot number were downloaded. We also acknowledge the Solar Influences Data Center (SIDC) for the compilation of the International Sunspot Number. NSO/Kitt Peak data used here were produced cooperatively by NSF/NOAO, NASA/GSFC, and NOAA/SEL. This study includes data about magnetic flux from the synoptic program at the 150-Foot Solar Tower of the Mt. Wilson Observatory and were kindly provided by J. Boyden. The Mt. Wilson 150-Foot Solar Tower is operated by UCLA, with funding from NASA, ONR, and NSF, under agreement with the Mt. Wilson Institute. The sunspot area data were compiled by D. Hathaway of NASA's Marshall Space Flight Center. J. Terradas thanks the Spanish Ministry of Science and Education for the funding provided by a Juan de la Cierva fellowship.

\section{References}

Ataç, T., \& Özgüç, A. 1996, Sol. Phys., 166, 201

Ataç, T., \& Özgüç, A. 1998, Sol. Phys., 180, 397

Carbonell, M., Oliver, R., \& Ballester, J. L. 1993, A\&A, 274, 497

Garcia, H. 1990, Sol. Phys., 127, 185

Gleissberg, W. 1947, Publ. Istanbul University Observatory, 31

Joshi, A. 1995, Sol. Phys., 157, 315

Joshi, B., \& Joshi, A. 2004, Sol. Phys., 219, 343

Howard, R. 1974, Sol. Phys., 38, 59

Knaack, R., Stenflo, J. O., \& Berdyugina, S. V. 2004, A\&A, 418, L17

Knoška, S. 1985, Contrib. Astron. Obs. Skalnate Pleso, 13, 217

Larson, H. J. 1982, Introduction to Probability Theory and Statistical Inference, 3rd Ed. (New York: John Wiley and Sons, Inc.)

Leftus, V. 1960, Bull. Astron. Inst. Czech, 11, 31

Li Kejun, Gu Xiaoma, Xiang Fuyuan, Liu Xiaohua, \& Chen Xuekun, 2000, MNRAS, 317, 897

Li, K. J., Schmieder, B., \& Li, Q. Sh. 1998, A\&AS, 131, 99

Li, K. J., Wang, J. X., Xiong, S. Y., et al. 2002, A\&A, 383, 648

Li, K. J., Wang, J. X., Zhan, L. S., et al. 2003, Sol. Phys., 215, 99

Li, K. J., Liu, X. H., Zhan, L. S., et al. 2005, New Astron., 8, 655

Newton, H. W \& Milson, A. S. 1955, MNRAS, 115, 398

Oliver, R., \& Ballester, J. L. 1994, Sol. Phys., 152, 481

Oliver, R., \& Ballester, J. L. 1995, Sol. Phys., 156, 145

Pulkkinen, P. J., Brooke, J., Pelt, J., \& Tuominen, I. 1999, A\&A, 341, L43

Rabin, D. M., DeVore, C. R., Sheeley, N. R., Harvey, K. L., \& Hoeksema, J. T. 1991, in Solar Interior and Atmosphere, ed. A. C. Cox, W. C. Livingston \& M. S. Matthews (The Universe of Arizona Press) 781

Reid, J. H. 1968, Sol. Phys., 52, 53

Roy, J. R. 1977, Sol. Phys., 5, 207

Schlamminger, L., 1991, Sol. Phys., 135, 407

Song, W. B., Wang, J. X., Ma, X. 2005, Chin. Astron. Astrophys., 29, 274

Swinson, D. B., Koyama, H., \& Saito, T. 1986, Sol. Phys., 106, 35

Temmer, M., Veronig, A., Hanslmeier, Otruba, W., \& Meserotti, M. 2001, A\&A, 375,1049

Temmer, M., Veronig, A., \& Hanslmeier, A. 2002, A\&A, 390, 707

Temmer, M., Rybák, J., Bendík, P., et al. 2006, A\&A, 447, 735

Verma, V. K. 1987, Sol. Phys. 114, 185

Verma, V. K. 1992, in The Solar Cycle, ed. K. Harvey, ASP Conf. Ser., 31, 429

Verma, V. K. 1993, ApJ, 403, 797

Verma, V. K. 2000, Sol. Phys., 194, 87

Vernova, E. S., Mursula, K., Tyasto, M. I., \& Baranov, D. G. 2002, Sol. Phys., 205,371

Vizoso, G., \& Ballester, J. L. 1990, A\&A. 229, 540

Waldmeier, M. 1957, ZAp, 43,149

Waldmeier, M. 1971, Sol. Phys., 29, 232

Wilson, R. M. 1987, Statistical Aspects of Solar Flares, NASA Technical Paper 2714

Yi, W. 1992, JRASC, 86, 2, 89 